\title{
Vorbemerkung: Über Künstlerbücher und Buchliteratur
}

Die Auseinandersetzung mit dem Buch und seiner Buchhaftigkeit (für die sich in der anglophonen Diskussion der Terminus ,bookness' etabliert hat; vgl. Teil A.1) verbindet das als eigenständige Kunstform seit den 1960er Jahren etablierte Künstlerbuch (artists' book, livre d'artiste) mit diversen Erscheinungsformen vor allem der neueren Literatur, welche man zusammenfassend deshalb als „Buchliteratur“ charakterisieren könnte, weil Form und Materialität des Buchs hier von konstitutiver Bedeutung für das jeweilige Werk sind. So sinnvoll es auch erscheint, terminologisch zu differenzieren: Zwischen künstlerischen und literarischen Buchwerken verläuft insgesamt keine klare Grenze; oft kommt es auf die Perspektive an, ob man ein Artefakt primär als buchkünstlerisches oder als literarisches Werk wahrnimmt und in entsprechende Traditionen und Kontexte einordnet. Auch sind wichtige Wegbereiter und Pioniere des Künstlerbuchs zugleich als literarische Autoren in Erscheinung getreten; genannt seien nur Stéphane Mallarmé, Dieter Roth und Raymond Queneau. Beide Bereiche ästhetischer Gestaltung sind auch insofern eng verbunden, als buchkünstlerische Werke vielfach im Zeichen der Bezugnahme auf Literarisches stehen: auf literarische Autoren, Gattungen oder Werke, auf Aspekte der Produktion und der Rezeption von Texten.

Gestalterische Auseinandersetzungen mit dem Buch verhalten sich in der Buchkunst und in der Literatur in vielem konvergent. Darin manifestiert sich hier wie dort ein ganzes Spektrum von Interessen, vor allem eines am Buch als einem prägenden Faktor von Kultur und als Medium kultureller Selbstdarstellung, an historischen Praktiken und Kommunikationsformen, die durch das Buch ermöglicht werden und durch spezifische Bücher geprägt sind.

Die 1960er und 1970er Jahre haben nicht allein eine intensive, breite und vielseitige künstlerische Arbeit an und mit dem Buch hervorgebracht; sie bieten zudem reiches Anschauungsmaterial zur Plausibilisierung der These, dass die jüngere Literaturgeschichte und die des Künstlerbuchs gemeinsam und auf ihre Verflechtungen hin betrachtet werden müssen. Text- und Literaturtheoretiker sowie Dichter und Schriftsteller haben wichtige Impulse für die Geschichte des Künstlerbuchs seit den 1960er Jahren gegeben, wie umgekehrt von Praktiken buchkünstlerischer Arbeit facettenreiche Anregungen auf literarische Autoren und ihre Projekte ausgegangen sind (und bis heute ausgehen). Auf theoretisch-reflexiver wie auf gestalterisch-explorativer Ebene sind die neuere Literatur und das neuere Künstlerbuch also durch eine gemeinsame Geschichte miteinander verbunden. Das experimentier- und innovationsfreudige spätere 20. Jahrhundert ist dabei zugleich eine besonders theoriefreudige Zeit. Schriftsteller und bildende Künstler nehmen intensiv an Diskursen über Kunst teil. Und vielfach kommt es zu einer Entdifferenzierung zwischen Theorie und Praxis, zwischen abstrakten Konzepten und konkreter ästhetischer Arbeit. 
So ergiebig es auch ist, durch Vergleiche ähnlich gestalteter Buchwerke selbst noch über konventionell gezogene Gattungsgrenzen (hier zwischen ,Literatur ${ }^{\star}$ und ,Kunst') hinaus verbindende Tendenzen, Interessen und Themen zu beobachten - das jeweils einzelne Werk sollte dabei doch stets auch als besonderes Gebilde mit besonderen Deutungsangeboten wahrgenommen werden. Dem wird im Folgenden durch den Rekurs auf zahlreiche Beispiele und ihre jeweiligen Eigenarten Rechnung getragen. 